\title{
Análise visual complexa: gráficos e diferenciabilidade de funções
}

Visual complex analysis: graphics and differentiality of functions

Francisco Regis Vieira Alves ${ }^{1}$; Mairton Cavalcante Romeu ${ }^{1}$

\section{RESUMO}

Reconhecidamente, determinados assuntos em Matemática, sobretudo aqueles do locus acadêmico, exigem um grau considerável de abstração mental. Neste artigo trazemos uma discussão do uso da tecnologia no contexto de um ramo em Matemática, não menos abstrato, chamado de Análise Complexa - AC. Deste modo, nos apoiando na perspectiva de Needham (2000), enfatizamos a interpretação gráfico-geométrica de determinados conceitos introdutórios, como de propriedades gráficas e o caráter de analiticidade das funções na variável complexa. Indicaremos que, a partir do uso de certas funções específicas e comandos dos softwares Geogebra e do CAS Maple, obtemos um cenário de aprendizagem que coloca em destaque a visualização como elemento impulsionador de um entendimento tácito e preliminar de situações vinculadas a cada conceito.

Palavras-chave: Visualização; Gráficos de funções; Análise Complexa.

\begin{abstract}
Admittedly, certain topics in Mathematics, especially those from academic locus, require a considerable degree of mental abstraction. In this article, we present a discussion of the use of technology in a branch of the Mathematics, no less abstract, called Complex Analysis - CA. Thus, supporting us in the perspective of Needham (2000), we emphasize the graphic-geometric interpretation of certain introductory concepts, such as the graphic properties and the character of the analyticity of the functions. We show that, from the use of a few specific functions and commands of the software Geogebra and CAS Maple, we obtain a learning scenario which highlights the visualization as an element that emphasize the understanding of situations related to each concept.
\end{abstract}

Keywords: Visualization; Graphs of the functions; Complex Analysis.

\footnotetext{
${ }^{1}$ IFCE - Instituto Federal de Educação, Ciência e Tecnologia do Ceará, Fortaleza/CE - Brasil.
} 


\section{INTRODUÇÃO}

No estudo em Análise Complexa - AC, recorrentemente, deparamos com o elevado grau de abstração e a mudança, em muitos casos considerável, de significação de determinados conceitos e processos matemáticos. Basta observar, por exemplo, os processos matemáticos de diferenciação e integração em uma variável complexa. Usualmente, denotamos por $w=f(z)=u(z)+i v(z)=\operatorname{Re}(f(z))+i \operatorname{Im}(f(z))$ para indicar a parte real e a parte imaginaria de uma função. Needham (2000, p. 56) explica que "obteremos um ganho de considerável insight relativo a uma função $f$ pelo desenho de figuras mostrando seu efeito sobre pontos, curva e formas. Todavia, seria muito bom se pudéssemos obter o comportamento simultâneo de $f$ para todos os valores de $z^{\prime \prime}$.

Ora, Needham (2000) aponta claramente este e outros entraves no estudo de AC, quando negligenciamos as possibilidades e potencialidades da tecnologia, com vistas à exploração do componente visual e perceptual. Neste sentido, mostrar-se-á que, com a exploração dos softwares Geogebra e do CAS Maple, conseguimos descrever, do ponto de vista gráfico-geométrico, o comportamento de $f$, para todos os valores complexa da variável ' $z$ ', como assim indica este autor no pequeno excerto assinalado. Doravante, discutiremos algumas propriedades no âmbito da AC, significadas a partir do uso da tecnologia.

\section{FUNÇÕES EM UMA VARIÁVEL COMPLEXA E VISUALIZAÇÃO}

Sabemos que, um dos métodos para se analisar o gráfico de uma função $f(x)$, em uma variável real, consiste em inspecionarmos, por exemplo, o comportamento de uma curva, no plano, para todos os valores de $x \in \operatorname{Dom}(f)$. Ou ainda, nos determos ao estudo dos pares ordenados $(x, f(x)) \in \mathbb{R}^{2}$. Não obstante, quando nos atemos ao estudo de funções em uma variável complexa $z$, tal abordagem não é mais praticável, uma vez que, para compreender o comportamento do par $(z, f(z))$ "precisamos de quadro dimensões. Duas para $z=x+i y$ e duas que correspondem $f(z)=u+i v^{\prime \prime}$ (NEEDHAM, 2000, p. 56).

Este autor adverte ainda que embora necessitemos de um espaço de quatro dimensões $\left(I R^{4}\right)$ para descrever o conjunto dos pontos $(x, y, u, v)=(z, f(z))$, o "gráfico, ele próprio é bidimensional, significando que apenas dois números reais são necessários para identificar cada ponto nele presente." (NEEDHAM, 2000, p. 56). Na figura 1, enfatizamos um modo de vislumbrar certos gráficos de funções do tipo $w=f(z)$. Neste sentido, "a imagem $f(z)$ de um ponto $z$ pode ser descrito por meio de sua distância $|f(z)|$, a partir da origem, e do ângulo $\arg [f(z)]$ formado com o eixo Ox." (NEEDHAM, 2000, p. 56-57). O autor exemplifica sua abordagem, por exemplo, no caso das funções $f(z)=z$ e $f(z)=z^{2}$. Nos casos abaixo, observamos o comportamento das superfícies no espaço, determinadas por $|z|$ e $|z|^{2}$, respectivamente.

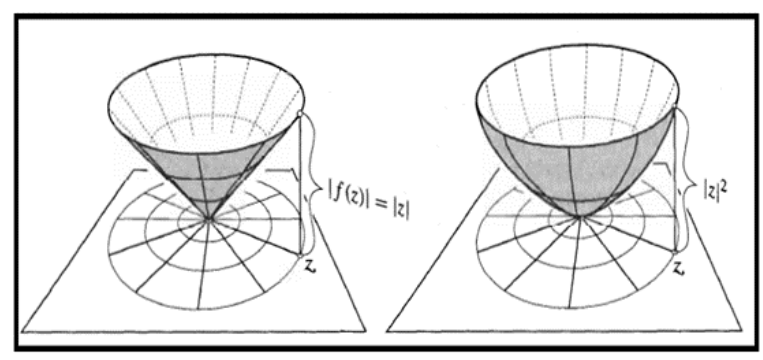

Figura 1. Método proposto por Needham (2000, p. 56-57) para visualização de gráficos de funções na variável complexa no contexto do espaço tridimensional. 
Vale salientar outras formas mais sofisticadas de se considerar os objetos indicados há pouco. Neste sentido, "de modo intrínseco, o gráfico de uma função complexa é simplesmente uma superfície bidimensional (superfície de Riemann) e que é suscetível para a visualização no espaço ordinário tridimensional.". (Idem, 2000, p. 56). De modo recorrente, Needham (2000) sugere o uso do computador com vistas à expansão da compreensão dos fenômenos matemáticos registrados no contexto da AC. Negligenciar o estado atual da tecnologia e suas potencialidades, possivelmente, permite relegar as tarefas de cunho intrincado dos estudantes "ao uso recorrente de algebrismos e um emprego restrito e pouco reflexivo de propriedades formais" (ALVES, 2011, p. 123). Deste modo, no próximo segmento, discutiremos algumas situações afetadas pela perspectiva sui generis de Needham (2000).

\section{EXEMPLOS NO ENSINO DE ANÁLISE COMPLEXA E O USO DE SOFTWARES}

Um dos nossos primeiros interesses nesta seção refere-se à identificação das raízes de funções polinomiais, do tipo $p(z)=a_{n} z^{n}+a_{n-1} z^{n-1}+\cdots+a_{2} z^{2}+a_{1} z+a_{0}$, onde $a_{n}, a_{n-1}, \ldots, a_{2}, a_{1}, a_{0} \in \mathbb{R}$. No trabalho de Perez (2012), encontramos a proposição de dois métodos que exploram a visualização das soluções de gráficos de funções polinomiais na variável complexa. De fato, a autora desenvolve em sua dissertação o estudo de superfícies modulares e, em seguida, o estudo de campos vetoriais. Esta última forma de abordagem no contexto da Física também é desenvolvida por Needham (2000, p. 450).

Na Figura 2, exemplificamos o comportamento esperado de funções na variável real, a partir da consideração de seus valores reais. Apontaremos, pois, a possibilidade de visualização, quando nos atemos a funções do tipo $f(x)=x^{2}+x+1$ e $f(z)=z^{2}+z+1$. Com base na figura 2, indicaremos um comportamento semelhante no comportamento da superfície determinada pela função $|f(z)|=\left|z^{2}+z+1\right|$ (Figura 2).

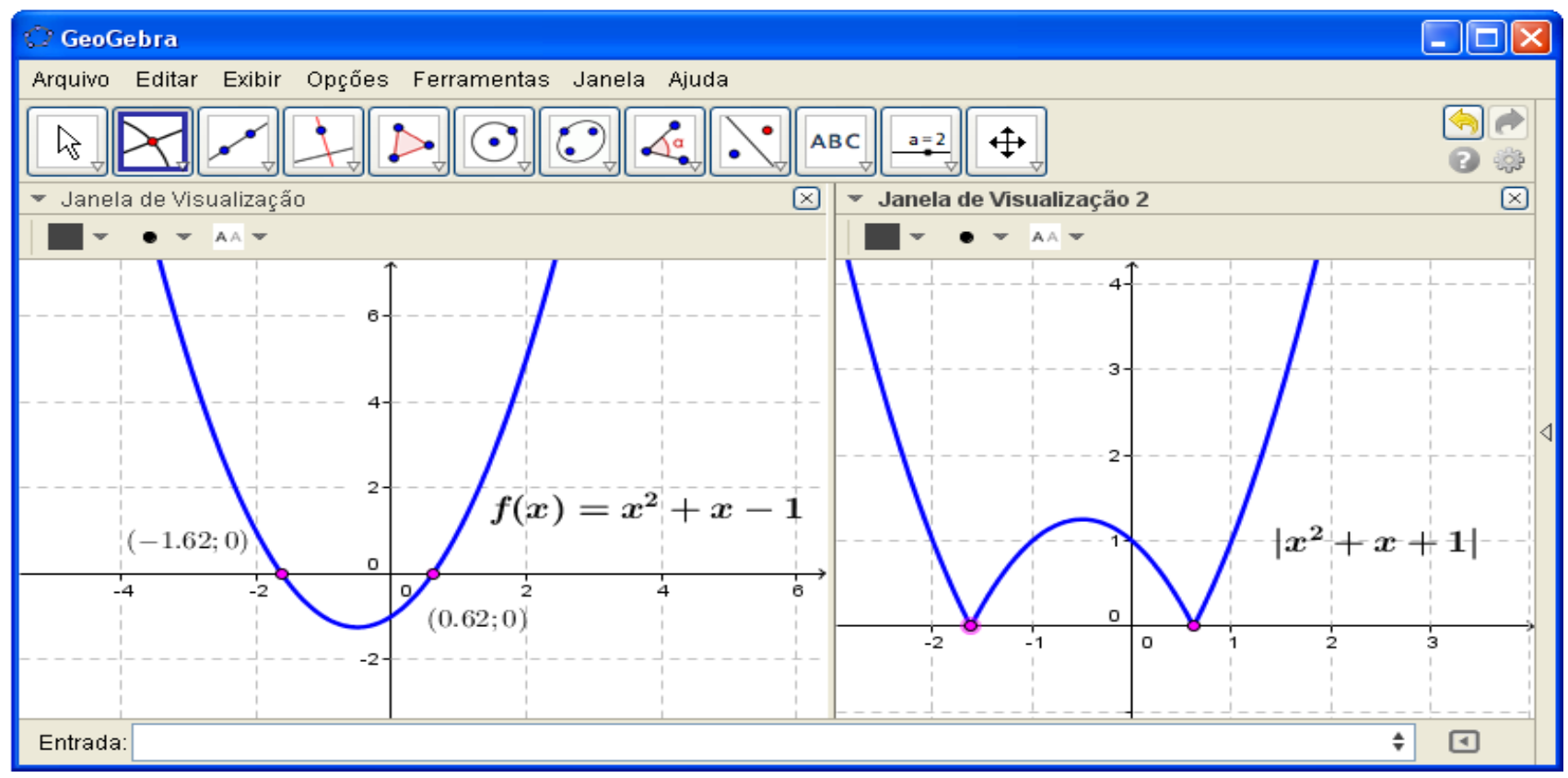

Figura 2. Processo discutido por Perez (2012) na visualização de raízes de funções polinomiais e sua comparação com o uso do software GeoGebra, de modo restrito na variável real.

Fonte: elaboração dos autores 


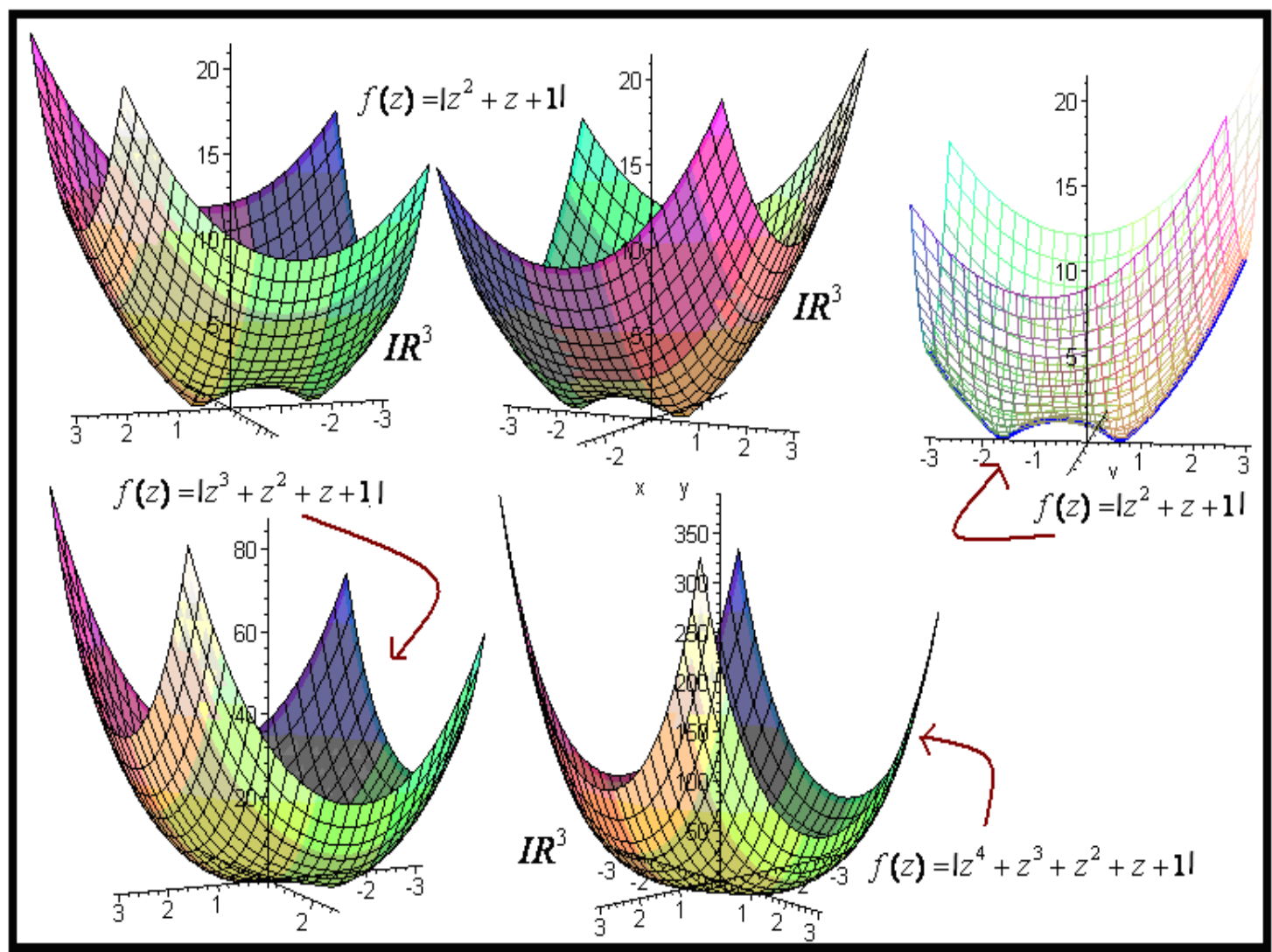

Figura 3. Descrição das superfícies associadas à visualização de funções modulares complexas Fonte: elaboração dos autores

Na Figura 3, consideramos as expressões polinomiais $z^{2}+z+1, z^{3}+z^{2}+z+1, z^{4}+z^{3}+z^{2}+z+1$. Enquanto que na figura 4 , acrescentamos o comportamento de $z^{5}+z^{4}+z^{3}+z^{2}+z+1$, $z^{6}+z^{5}+z^{4}+z^{3}+z^{2}+z+1$ e $z^{7}+z^{6}+z^{5}+z^{4}+z^{3}+z^{2}+z+1$. Destacamos, todavia, que na figura 4 colocamos em evidência o comportamento das curvas de nível (no plano), determinadas pela parte real da listagem acima, uma vez que, ao considerarmos seus valores absolutos, as partes imaginárias serão todas nulas.

Registramos o padrão gráfico-geométrico obtido por meio do CAS Maple (ver Figura 4). Observamos que na Figura 3, adotamos a abordagem sugerida por Needham (2000, p. 56). Vale acentuar que 0 grau de cada função polinomial corresponderá ao número de vizinhanças, no plano, indicativo de cada raiz complexa. Trazemos, então, a explicação de Perez $(2012$, p. 6) ao declarar que "superfícies modulares apresentam pontos inferiores (de mínimo) tocando o plano complexo em dois pontos.".

Acentuamos ainda que o Teorema Fundamental da Álgebra - TFA diz que um polinômio de grau n, com coeficientes complexos, possui exatamente $n$ raízes, levando-se em consideração suas multiplicidades. Ora, podemos fornecer uma demonstração mais elementar, de natureza geométrica para este teorema (SANTOS, 1998, p. 7-8). Por outro lado, do ponto de vista computacional, empregamos o mesmo princípio de Santos (1998) que permite verificar (visualizar) o TFA com o CAS Maple.

Perez (2012, p. 6-7) parte do caso de funções polinomiais do $2^{\circ} \mathrm{grau}$. Argumenta que "desde que um número complexo é zero se, e somente se, seu módulo é também zero, os zeros das funções serão os pontos em que os pontos da superfície modular entram em contato com o plano complexo.". Podemos, agora, comparar as figuras 3 e 4, notando que as vizinhanças determinadas pelas curvas 
de nível na figura 4 indicam o "toque" da superfície no plano complexo e, então, a localização de uma raiz de $p(z)$.

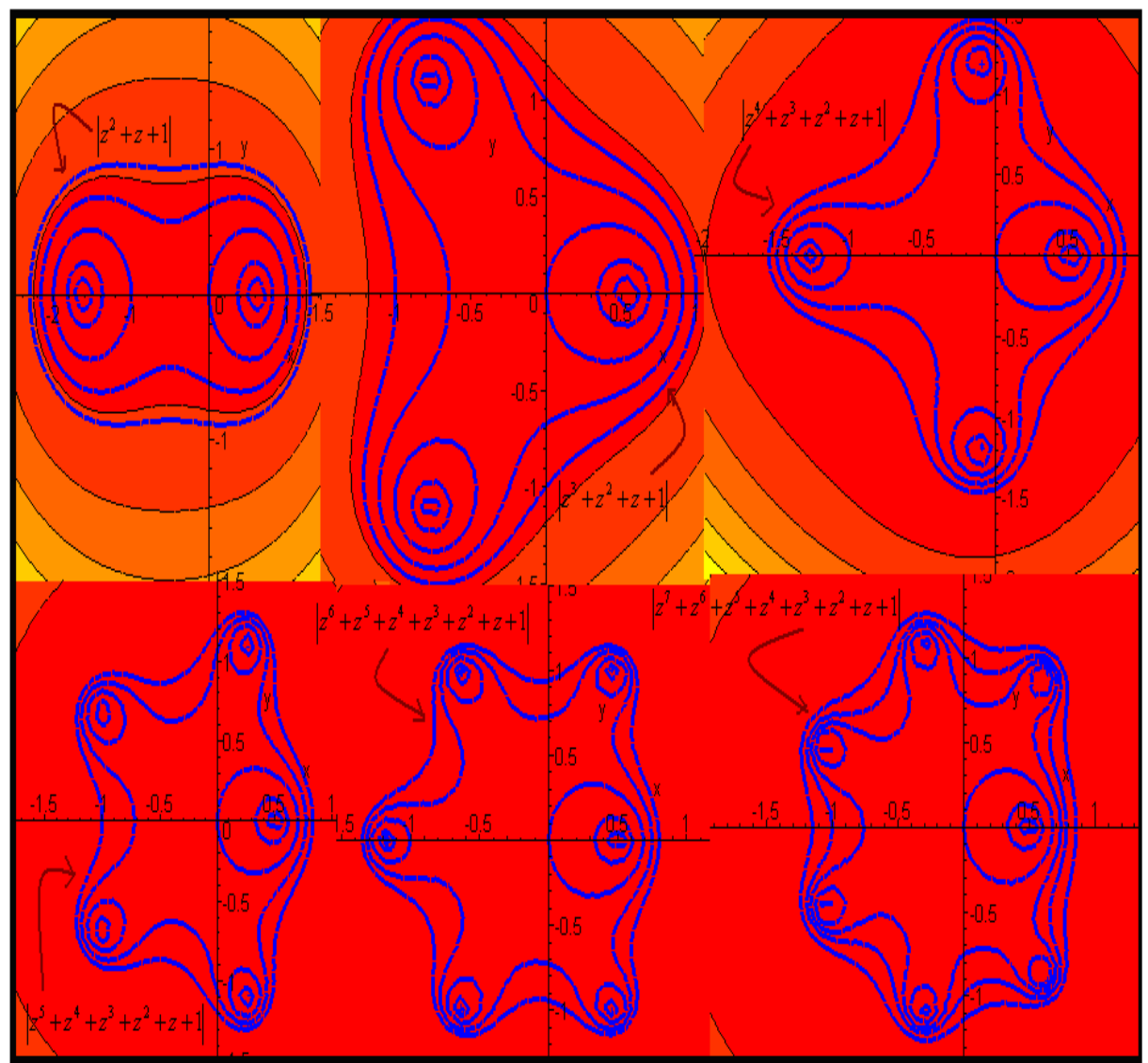

Figura 4. Descrição/visualização das curvas de nível a partir da consideração do módulo das funções em uma variável complexa com o auxílio computacional (CAS - Maple)

Fonte: elaboração dos autores

Nosso segundo exemplo pode ser visto em Neto (1993, p. 58), quando encontramos a seguinte expressão $f^{\prime}\left(z_{0}\right)=\lim _{h \rightarrow 0} \frac{f\left(z_{0}+h\right)-f\left(z_{0}\right)}{h}(*)$ que indica a derivada, no ponto $z=z_{0}$, de uma função contínua e diferenciável, definida em que $U$ é um conjunto do plano. Ora, por intermédio de uma apreciação açodada, pode não parecer muito claro ao leitor declarar que $f^{\prime}\left(z_{0}\right)$ é de fato um número complexo. Ademais, apesar da análise matematicamente irretocável de Lins (1993), questionamos: que outros significados, sobretudo os de ordem gráfico-geométrica, poderiam ser agregados a tal definição, com o intuito de proporcionar um significado intuitivo a este conceito?

Apoiar-nos-emos no raciocínio comparativo estabelecido por Needham (2000, p. 194-195) para os casos real e complexo. Preliminarmente, este autor salienta que

No cálculo ordinário em variável real nós possuímos um potente meio de visualização da derivada $f^{\prime}$ de uma função de definida da reta para reta real, chamado de declividade do gráfico. Infelizmente, devido à nossa deficiência de imaginação em quarta dimensão, não podemos desenhar o gráfico de uma função complexa, e assim não podemos generalizar esta concepção particular de derivada por meio de um caminho óbvio. (NEEDHAM, 2000, p. 194).

Um primeiro argumento apresentado pelo autor, com vistas ao entendimento da definição formal $(*)$, consiste em separar e considerar duas cópias do espaço unidimensional, em posição paralela (ver 
figura 5-b). De modo antecipado, ambos os eixos Ox serão tomados como eixos reais no plano complexo. Depois, observa que o número indicado por $\left|f^{\prime}(x)\right|$ descreve o modo pelo qual o vetor $x$ deve ser expandido para obter sua imagem $f(x)$. Todavia, algebricamente, " $f^{\prime}(x)$ é o número real tal que deve multiplicado ao valor inicial $d(x)$ para obter sua imagem" (ver Figura 6, na parte superior).

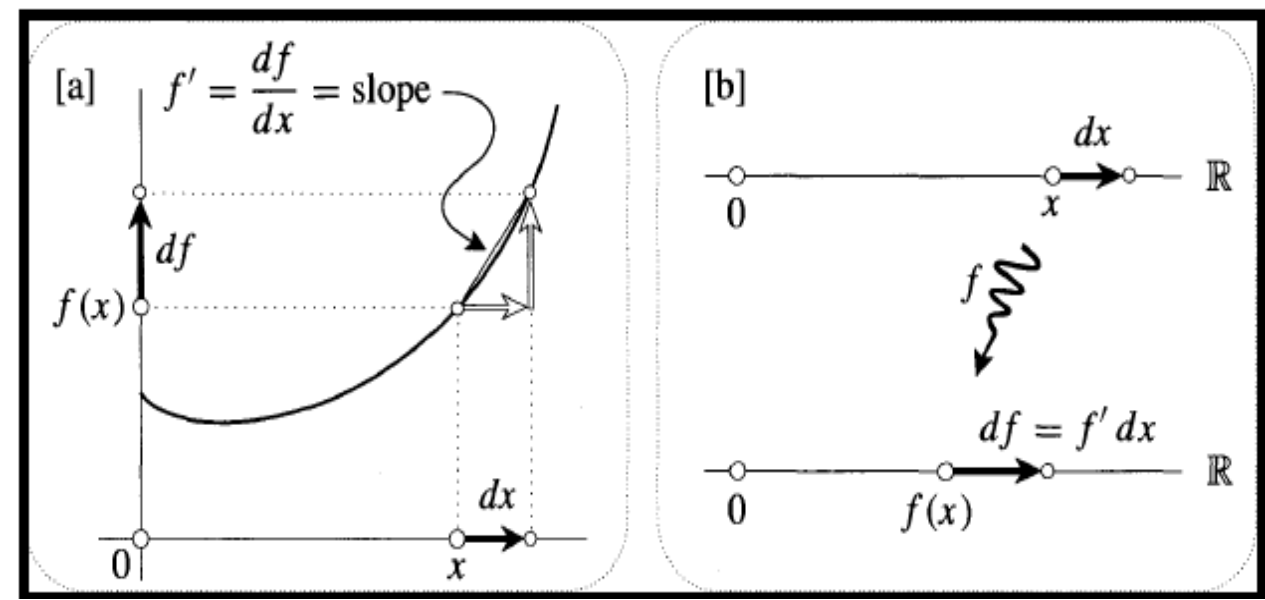

Figura 5. Needham (2000, p. 195) estabelece um argumento para descrever de modo geométrico a derivada ordinária de funções em uma variável real.

Na parte superior da Figura 6, Needham (2000, p.195) adota um argumento mnemônico afim de significar/exprimir a ação da derivada no caso real. Na figura 6, o autor explica que "se $f^{\prime}(x)>0$ então a imagem do elemento positivo $d x$ é um positivo $d f$, porém, se $f^{\prime}(x)<0$ então a imagem infinitesimal do vetor $d f$ é negativo e aponta para o sentido esquerdo.". Neste último caso, o elemento $d f$ (ver fig. 6 ao lado direito) pode ser obtido e interpretado por meio da expansão de $d x$ pelo fator $\left|f^{\prime}(x)\right|$, então a rotação do mesmo por um ângulo de $\pi \mathrm{rad}$.

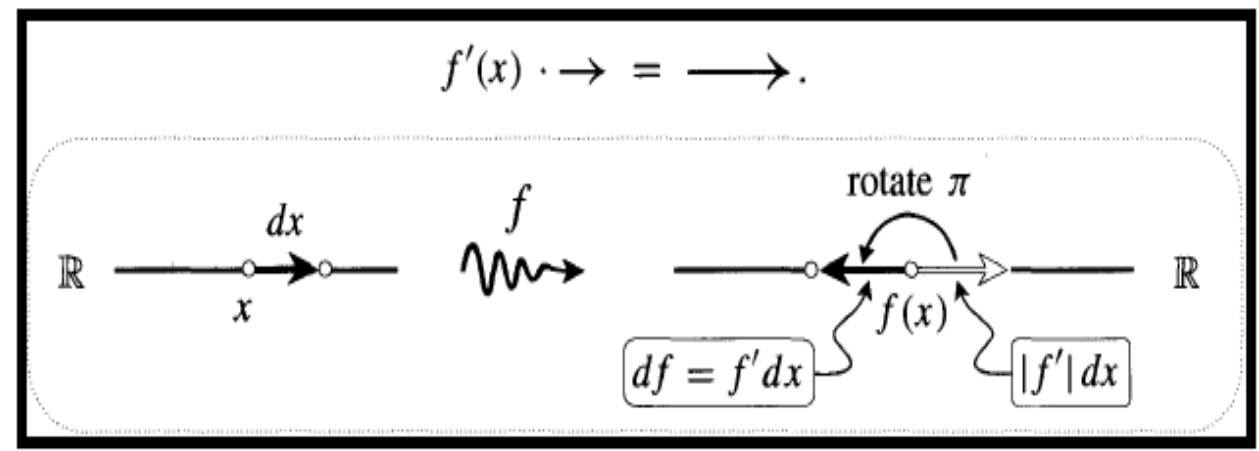

Figura 6. Needham (2000, p. 195) fornece uma descrição heurística para a noção de derivada em $\mathbb{R}$

Reparemos, nesse caso, que se tratando de uma variável real, multiplicamos por um fator real e efetuamos uma rotação de $0^{\circ}$ ou $\pi$. Mais adiante, ele adota um raciocínio semelhante e generalizado para significar funções do tipo $f(z)=f(x+i y)$. Quando Needham (2000, p.195) se refere à classe de funções na variável complexa, acentua que "para transformar a seta de cor branca em outra seta de cor preta, não apenas uma expansão é necessária, como também, uma rotação.". (ver Figura 7). 


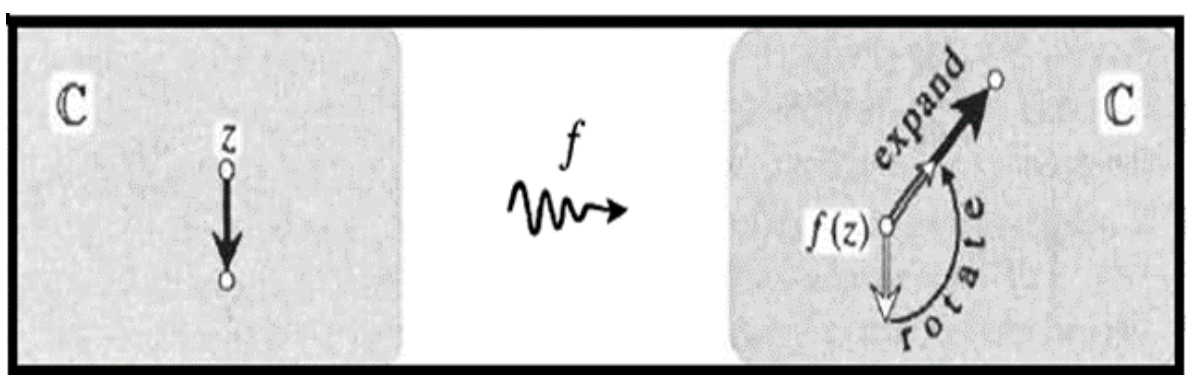

Figura 7. Needham (2000, p. 196) estabelece um argumento para descrever de modo geométrico a derivada de funções na variável complexa

Na Figura 7, ao considerar uma função arbitrária, o autor indicou o significado do processo de derivação, exemplificando com o seguinte exemplo, quando menciona que ao multiplicarmos a sete (vetor) de cor branca por 2 e, em seguida, rotacionarmos por $\frac{3 \pi}{4}$ (figura 7). Fato que contrasta com o caso de uma função, em uma variável real, que indicamos por $f^{\prime}(x)$, em que o único ângulo de rotação é 0 ou $\pi$ (figura 6 ). Por fim, "a derivada complexa $f^{\prime}(z)$ pode ser agora introduzida como um número complexo pelo qual multiplicamos um número infinitesimal z." (NEEDHAM 2000, p. 196).

Apontamos na figura 8, no canto superior, uma figura mnemônica que pode ser interpretada/descrita por intermédio da seguinte ilação: "A fim de produzir um efeito correto, o comprimento de $f^{\prime}(z)$ deve ser o fator de magnificação, e o argumento de $f^{\prime}(z)$ deve ser o ângulo de rotação." (Idem, 2000, p. 196).

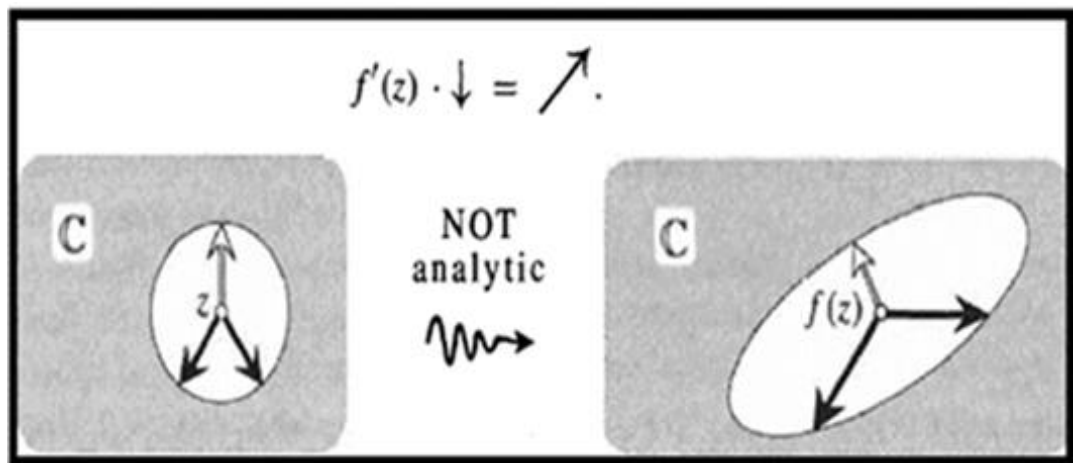

Figura 8. Needham (2000, p. 196) descreve a ação ou processo matemático resultante da derivação em $\mathbb{C}$ e o comportamento de funções não analíticas

Atentamos para um fato assinalado por ele quando menciona que "para encontrar $f^{\prime}(z)$ temos considerado a imagem de uma seta específica em $z$, porém (diferentemente do caso unidimensional $\mathbb{R}$ ) existem agora infinitas possibilidades de direção para tais setas.". (NEEDHAM, 2000, p. 196). O cuidado do autor na descrição, por intermédio de figuras e desenhos que transmitem a ideia de um processo complexo como este, do ponto de vista formal, deve ser reconhecido e, tal entendimento pode ressignificar, agora, a definição formal que indicamos anteriormente em (*).

Em consonância do estudo da derivada no caso da variável real, Needham (2000, p. 197) observa que "devemos ressaltar, entretanto, que todas as nossas recentes ilustrações são concernentes a propriedades locais, e deste modo com setas e figuras infinitesimais.". Por fim, esclarece ao leitor a introdução de uma terminologia particular e exclusiva em sua abordagem, quando assinala ainda que "introduzimos o termo 'amplitwist' (em adição ao termo derivada) porque o mesmo é sugestivo, e porque o mesmo propicia um raciocínio mais simples de se explicar. Todavia, o estudante que encontra este assunto pela primeira vez deve ficar atento que "em todos os outros livros a palavra derivada é usada." (NEEDHAM, 2000, p. 198). 
Para concluir, vamos admitir que num ponto $z_{0}=x_{0}+i y_{0}$, exista $f^{\prime}\left(z_{0}\right)$. Um argumento padrão é tomar as seguintes possibilidades de se aproximar de $z_{0}$, por meio de $z_{1}=x+i y_{0}$ e $z_{2}=x+i y_{0}$. Ora, sabendo que $f(z)=u(x, y)+i v(x, y)$, escrevemos:

$$
\frac{f(z)-f\left(z_{0}\right)}{z-z_{0}}=\frac{u\left(x, y_{0}\right)-u\left(x_{0}, y_{0}\right)}{x-x_{0}}+i \frac{v\left(x, y_{0}\right)-v\left(x_{0}, y_{0}\right)}{x-x_{0}}
$$

Passando o limite $z_{1} \rightarrow z_{0}$, obteremos que: $f^{\prime}\left(z_{0}\right)=\frac{\partial u}{\partial x}\left(x_{0}, y_{0}\right)+i \frac{\partial v}{\partial x}\left(x_{0}, y_{0}\right)$. Com base no mesmo argumento, tomando $z_{2} \rightarrow z_{0}$, inferimos que: $f^{\prime}\left(z_{0}\right)=-i \frac{\partial u}{\partial y}\left(x_{0}, y_{0}\right)+\frac{\partial v}{\partial y}\left(x_{0}, y_{0}\right)$. Dado que existe $f^{\prime}\left(z_{0}\right)$, concluiremos que: $\left\{\frac{\partial u}{\partial x}\left(x_{0}, y_{0}\right)=\frac{\partial v}{\partial y}\left(x_{0}, y_{0}\right), \frac{\partial v}{\partial x}\left(x_{0}, y_{0}\right)=-\frac{\partial u}{\partial y}\left(x_{0}, y_{0}\right)(* *)\right.$.

Ora, as condições (**) conhecidas como equações de Cauchy-Riemann (LINS, 1993, p. 59), estão presentes (em sua forma analítica) em qualquer compêndio especializado sobre AC. Não obstante, que possíveis interpretações gráfico-geométricas podem ser extraídas das expressões em (**)? Quais relações conceituais e possíveis ligações podemos realizar com o Cálculo Diferencial em várias variáveis?

Com o intuito de indicar uma via positiva para os dois questionamentos acima, escolhemos duas funções designadas por $f(z)=\frac{1}{z}$ e $g(z)=\frac{z^{3}+2 z+1}{z^{3}+1}$. Vamos, então, buscar a descrição das condições das equações de Cauchy-Riemann, do ponto de vista geométrico. De fato, com o auxílio do CAS Maple, listamos as derivadas parciais de $1^{\text {a }}$ ordem de $f(z)=\frac{1}{z}=\frac{x}{x^{2}+y^{2}}-i \frac{y}{x^{2}+y^{2}}$, do seguinte modo:

$$
\frac{\partial}{\partial x}\left(\frac{x}{x^{2}+y^{2}}\right)=\frac{1}{x^{2}+y^{2}}-\frac{2 x^{2}}{\left(x^{2}+y^{2}\right)^{2}} \quad \frac{\partial}{\partial y}\left(-\frac{y}{x^{2}+y^{2}}\right)=-\frac{1}{x^{2}+y^{2}}+\frac{2 y^{2}}{\left(x^{2}+y^{2}\right)^{2}}
$$

Deparamos maiores dificuldades no caso da descrição das partes real e imaginária de $g(z)=\frac{z^{3}+2 z+1}{z^{3}+1}=u(x, u)+i v(x, y)$. Com o software, escrevemos:

$u:=(x, y) \rightarrow \frac{\left(x^{3}-3 x y^{2}+2 x+1\right)\left(x^{3}-3 x y^{2}+1\right)}{\left(x^{3}-3 x y^{2}+1\right)^{2}+\left(3 x^{2} y-y^{3}\right)^{2}}+\frac{\left(3 x^{2} y-y^{3}+2 y\right)\left(3 x^{2} y-y^{3}\right)}{\left.\left(x^{3}-3 x y^{2}+1\right)\right)^{2}+\left(3 x^{2} y-y^{3}\right)^{2}}$

$u:=(x, y) \rightarrow \frac{\left(3 x^{2} y-y^{3}+2 y\right)\left(x^{3}-3 x y^{2}+1\right)}{\left(x^{3}-3 x y^{2}+1\right)^{2}+\left(3 x^{2} y-y^{3}\right)^{2}}-\frac{\left(x^{3}-3 x y^{2}+2 x+1\right)\left(3 x^{2} y-y^{3}\right)}{\left(x^{3}-3 x y^{2}+1\right)^{2}+\left(3 x^{2} Y-y^{3}\right)^{2}}$

Daí, vislumbramos, do ponto de vista analítico, os entraves relativos à obtenção, em termos das variáveis $\mathrm{x}$ e $\mathrm{y}$, das partes real, que indicamos por $\mathrm{u}(\mathrm{x}, \mathrm{y})=\operatorname{Re}(f(z))$ e imaginária $\mathrm{v}(\mathrm{x}, \mathrm{y})=\operatorname{Im}(f(z))$ da função $f(z)$ (sem o uso do software). Por outro lado, com o auxílio do CAS Maple, obtemos facilmente as expressões correspondentes de $u(x, y), v(x, y)$ e, além disso, podemos comprovar, do ponto de vista gráfico-geométrico, as equações $(* *)$. Na figura 9 , observamos que fora da origem, as 
superfícies (na cor verde e vermelho) coincidem. No ponto $(0,0)$ divisamos, entretanto, certos "vulcões" ou "picos", indicando imagens ilimitadas neste ponto.

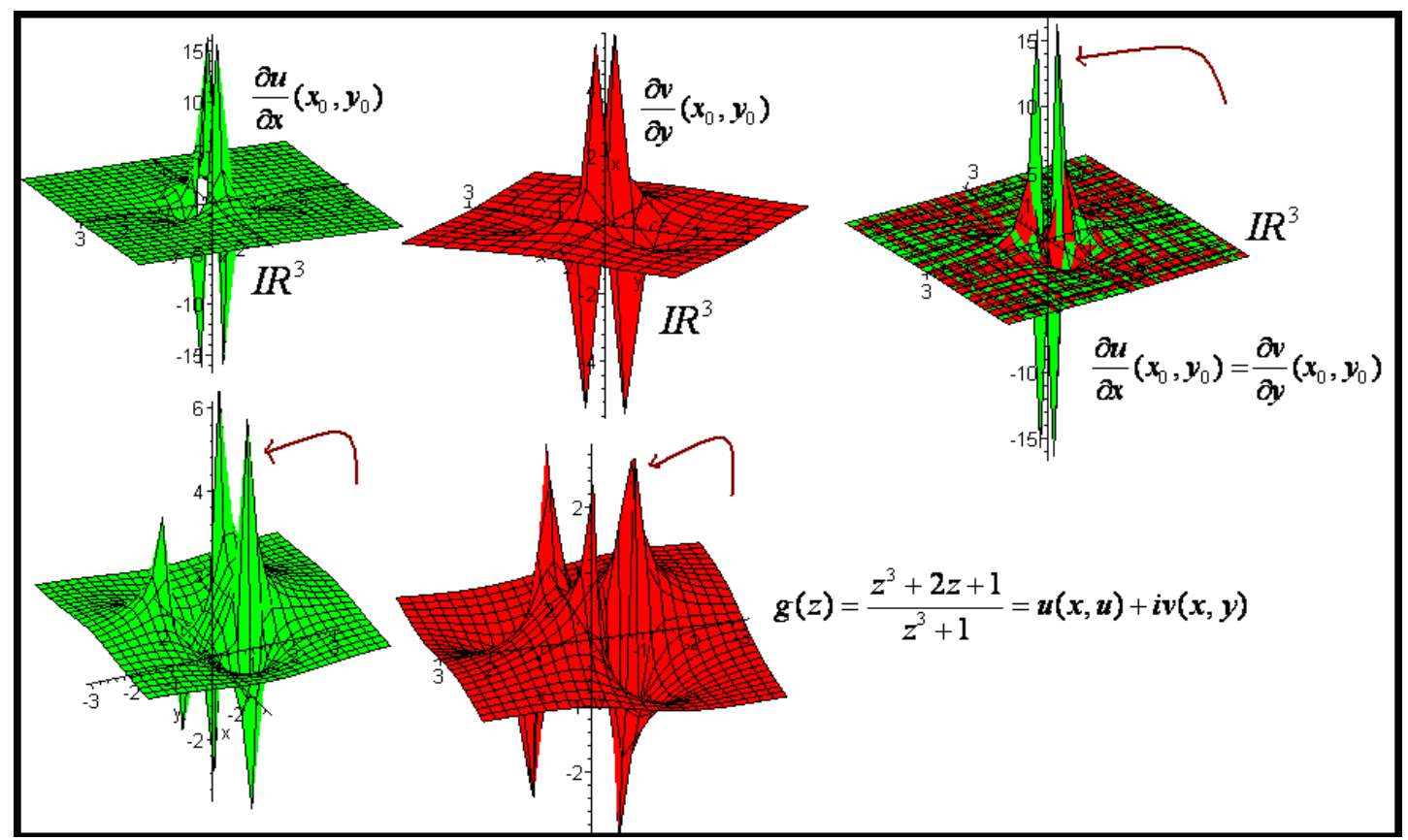

Figura 9. Visualização das derivadas indicadas nas condições de Cauchy-Riemann e das partes real e imaginária de $g(z)$. Fonte: elaboração dos autores

Na Figura 9, no canto inferior, observamos três picos correspondentes a cada raiz da expressão $z^{3}+1=0$. Podemos ainda, com amparo na figura 10 , exibir as superfícies produzidas com o software, relativas às derivadas parciais $\frac{\partial u}{\partial x}\left(x_{0}, y_{0}\right) \mathrm{e} \frac{\partial v}{\partial y}\left(x_{0}, y_{0}\right)$. Reparemos que as mesmas coincidem para pontos $\left(x_{0}, y_{0}\right) \neq(0,0)$, como divisamos ao lado direito da fig. 10. Entretanto, o comportamento é imprevisível na origem.

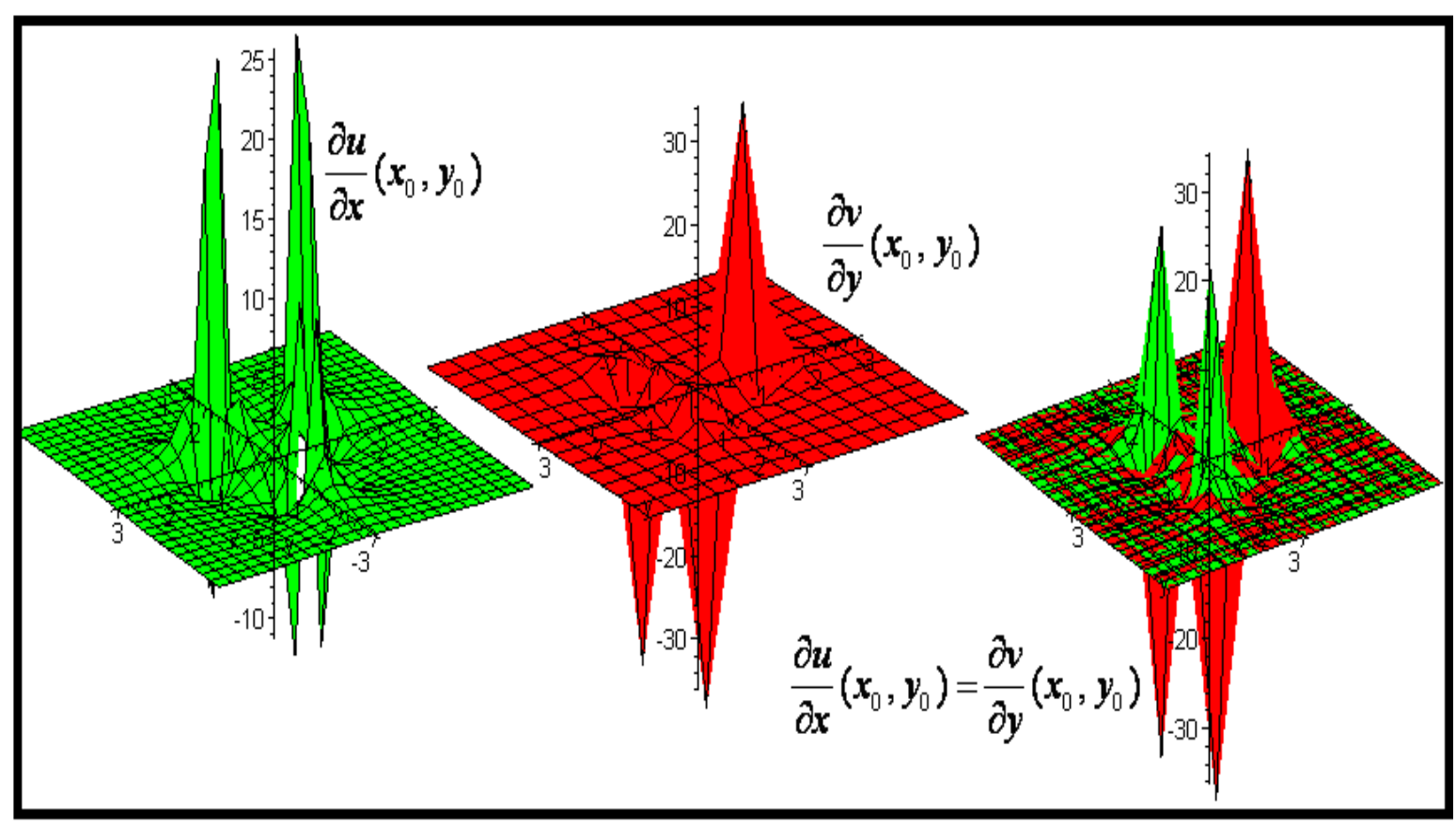

Figura 10. Visualização das derivadas indicadas nas condições de Cauchy-Riemann.

Fonte: elaboração dos autores 
Sublinhamos que na seguinte descrição $f^{\prime}\left(z_{0}\right)=\frac{\partial u}{\partial x}\left(x_{0}, y_{0}\right)+i \frac{\partial v}{\partial x}\left(x_{0}, y_{0}\right)$, o aluno terá a oportunidade de resgatar seu conhecimento sobre diferenciabilidade de funções na variável real. Ora, neste caso, do ponto de vista geométrico, a diferenciabilidade consiste em verificarmos a possibilidade de se obter uma aproximação local de uma função $z=f(x, y)$, por meio de um plano que tangencia seu gráfico num ponto. Tal plano é determinado pela expressão $T(x, y)=f\left(x_{0}, y_{0}\right)+\frac{\partial f}{\partial x}\left(x_{0}, y_{0}\right)\left(x-x_{0}\right)+\frac{\partial f}{\partial y}\left(x_{0}, y_{0}\right)\left(y-y_{0}\right)$. Por outro lado, a diferenciabilidade de uma função complexa num ponto corresponde aos acréscimos dos valores de uma função considerada, numa determinada vizinhança do ponto, em relação aos valores nesses pontos serem bem aproximados por uma função linear complexa. A aproximação, semelhantemente ao caso real, pode ser considerada boa, na medida em que os erros da aproximação tenderem, pois, para zero.

Para concluir, recordamos que Shokranian (2011, p. 136) acentua as relações conceituais entre funções analíticas e funções harmônicas. Na figura 11, ao lado direito, indicamos o empenho didático do autor em transmitir para o leitor a sensação visual esperada decorrente da propriedade das curvas de nível associadas a funções harmônicas. No lado esquerdo, trazemos as curvas associadas à função $f(z)=z^{3}+1$. Um caso trivial de não analiticidade é o exemplo de $f(z)=\bar{z}=x-i y$. Poderíamos ainda inspecionar a função $f(z)=z^{6}=\rho^{6}[\cos (6 \theta)+i \operatorname{sen}(6 \theta)]$, onde $\rho=|z|$. Nesse caso, obtemos facilmente que $\operatorname{Re}(f)=\rho^{6} \cos (6 \theta)$ e $\operatorname{Im}(f)=\rho^{6} \cos (6 \theta)$.

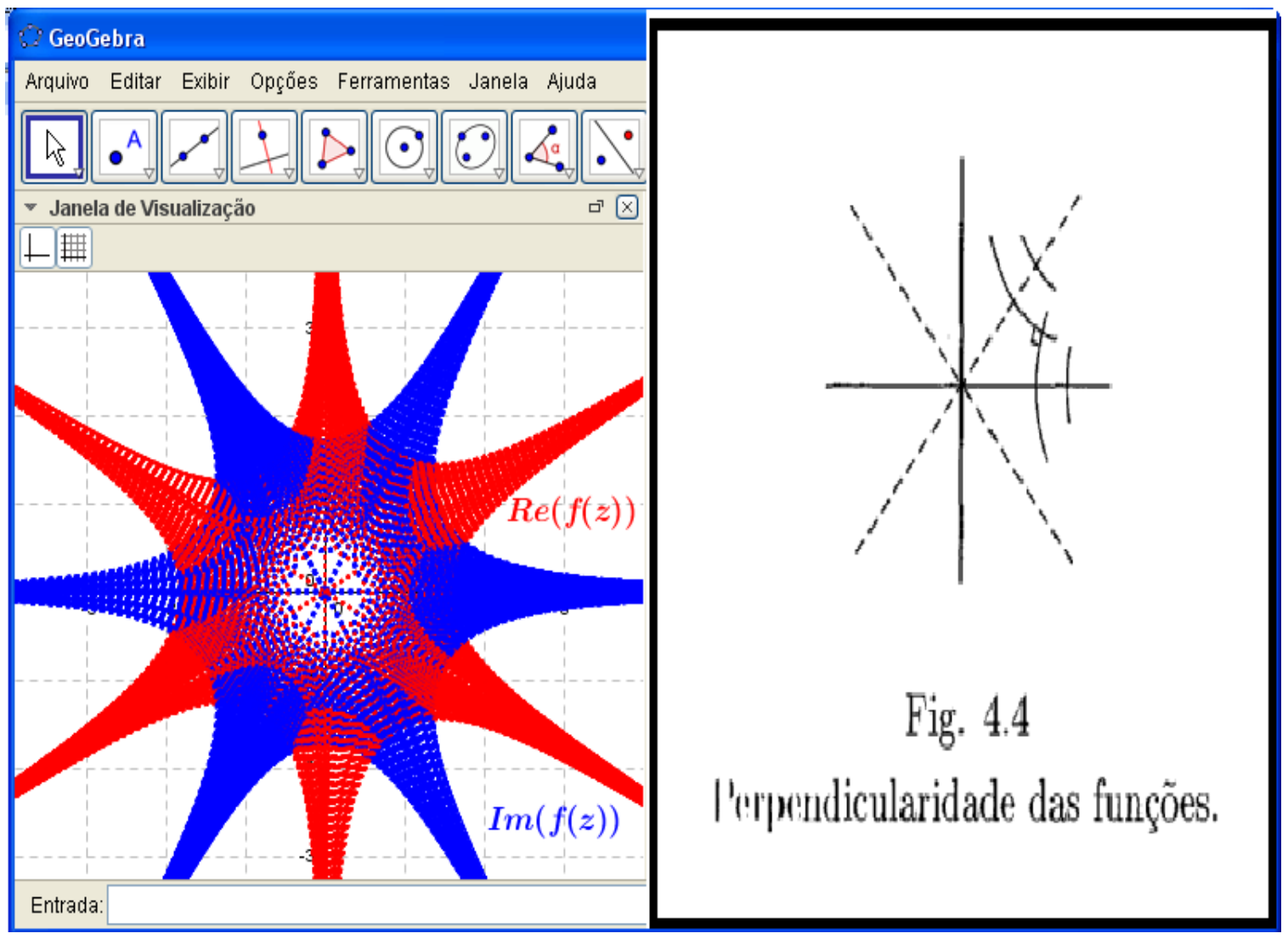

Figura 11. Relações conceituais e visuais entre funções analíticas e harmônicas.

Fonte: elaboração dos autores 


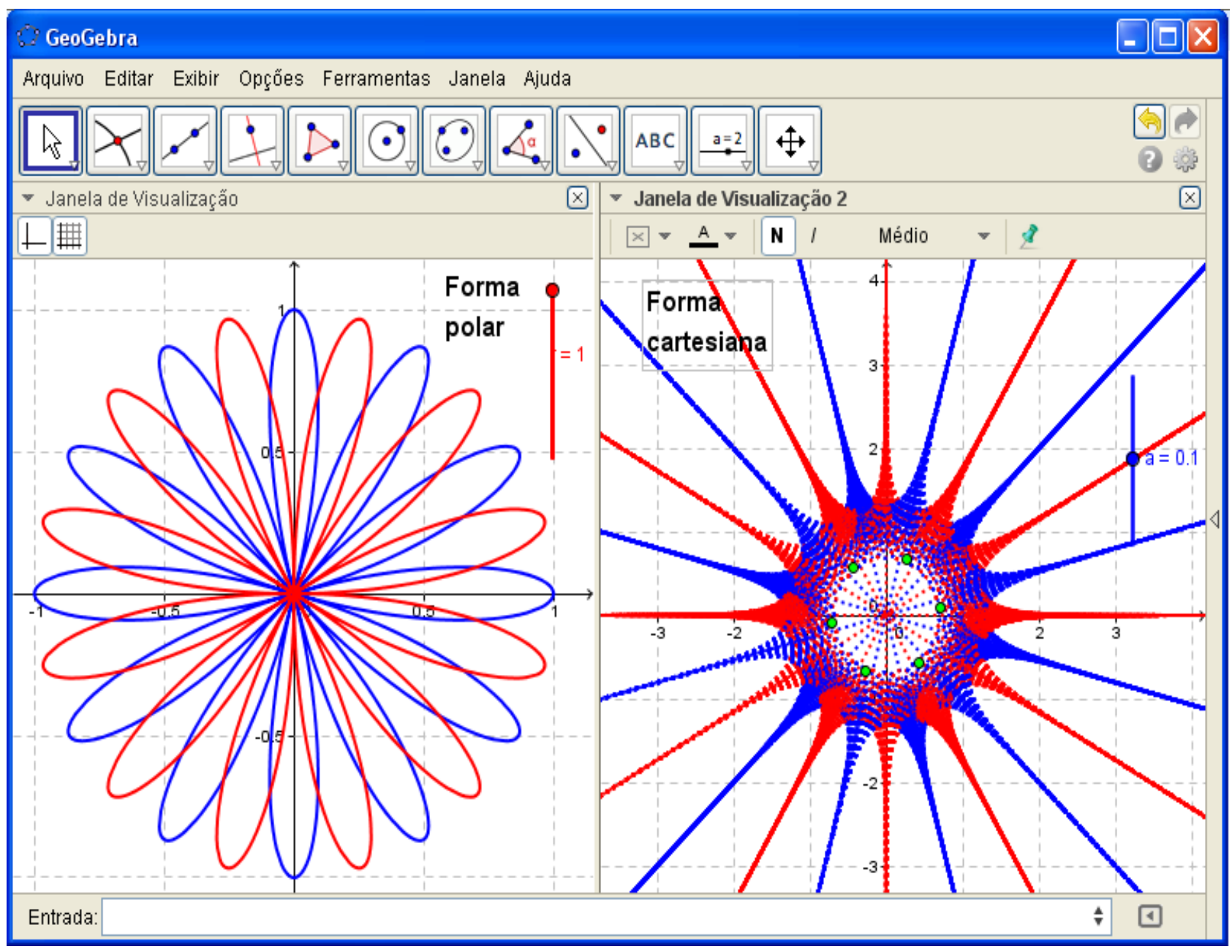

Figura 12. Mudança de coordenadas cartesianas para coordenadas polares.

Fonte: elaboração dos autores

Na Figura 12, efetivamos a mudança de coordenadas cartesianas (ao lado direito) para coordenadas polares (ao lado esquerdo, fig. 12). A limitação do software, neste caso, reside em determinar uma quantidade maior, relativa aos pontos de intersecção das curvas em coordenadas polares, como indicamos na função $f(z)=z^{6}=\rho^{6}[\cos (6 \theta)+i \operatorname{sen}(6 \theta)]$. Vale assinalar que apesar da mudança, relativa ao comportamento da curva em coordenadas polares, determinadas propriedades podem ser perdidas, como por exemplo, a possibilidade da determinação e localização de todas as suas seis raízes e o comportamento (relativo à perpendicularidade das funções) indicado na figura 11, ao lado direito, por Shokranian (2011, p. 136).

Para concluir, trazemos uma construção com o software Geogebra de acordo com os passos teóricoconceituais explicados por Needham (2000), nos parágrafos anteriores. Sabemos que na variável real, a derivada é descrita por $f^{\prime}(x) \cdot d x=d f$. No caso da variável complexa, designamos na figura 13 , uma variação correspondente de acréscimo $0 \leq r \leq 1$. Em seguida, determinamos um ponto móvel ' $z$ ' (em coordenadas no plano). Agora, reparemos que para determinar o comportamento da expressão $f^{\prime}(z) \cdot d z$. Reparemos que ao considerarmos a função $f(z)=z^{2} \therefore f^{\prime}(z)=2 z$. Na figura 13 , consideramos também uma translação do vetor ' $z$ ' por 'i' definido como Vetor $[(0, r)]$, obtendo-se o vetor $z^{\prime}$. Depois, avaliamos o componente $d z$ pelo vetor $\left[z, z^{\prime}\right]$. 


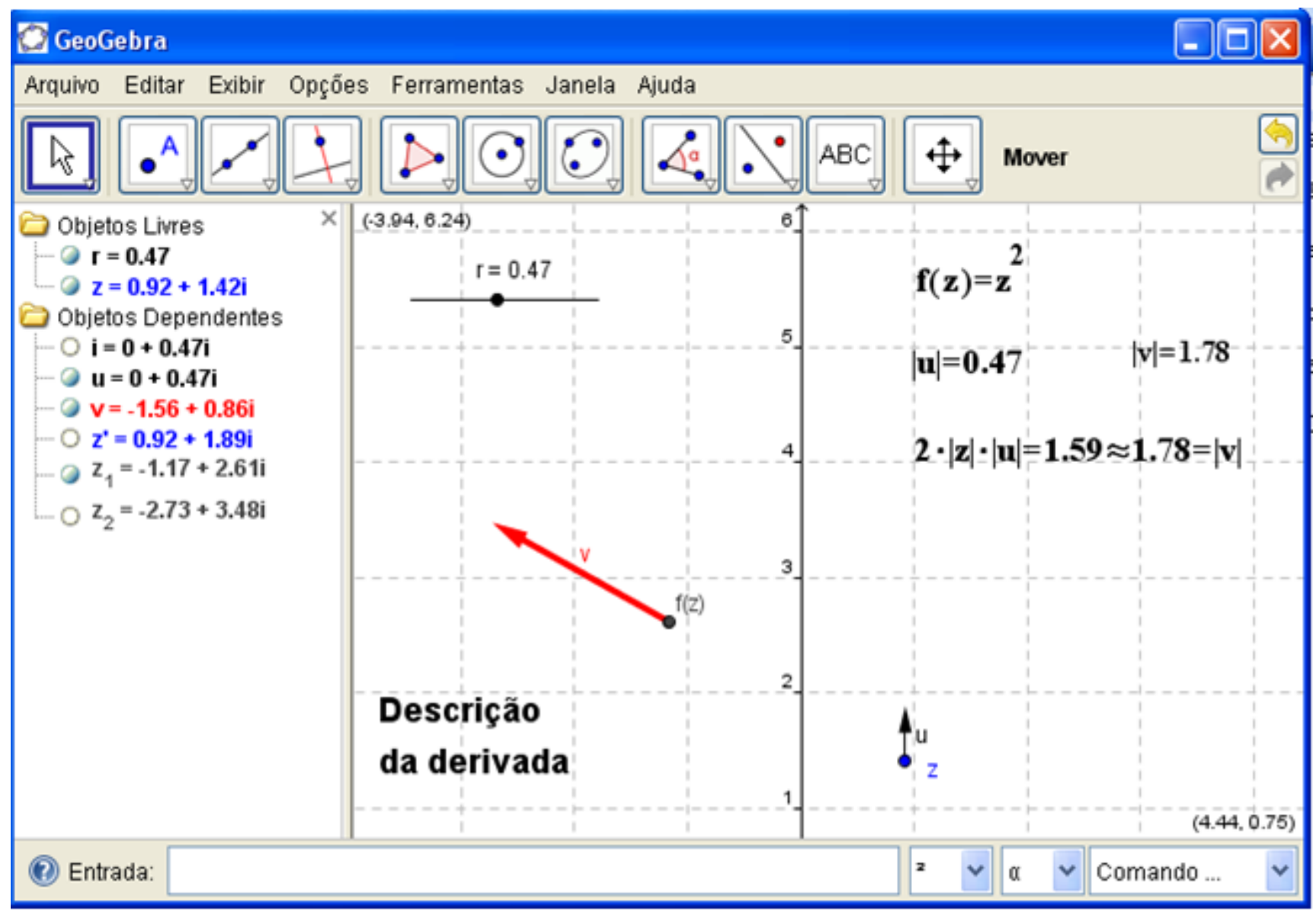

Figura 13. Descrição geométrica da noção de derivação de funções na variável complexa.

Fonte: elaboração dos autores

Na Figura 13, indicamos a expressão $2|z| \cdot|u|$ o que corresponde à expressão da derivada analítica. Podemos, agora, visualizar o resultado da ação do operador sobre um vetor dado preliminarmente. Mais geralmente falando, determinamos o efeito local de $f$ sobre um vetor infinitesimal $\Delta z$, pelo produto da magnificação $f^{\prime}(z)$ e o correspondente argumento deste vetor.

\section{CONCLUSÕES OU CONSIDERAÇÕES FINAIS}

Neste artigo buscamos enfatizar e descrever situações em que a visualização de determinadas propriedades e a exploração perceptual garantida pelo emprego da tecnologia no contexto do ensino (ALVES, 2012; 2013a; 2013b; 2015; 2016; 2017) permite atribuir significados intuitivos, tácitos e heurísticos a determinados conceitos abstratos no ramo da $\mathrm{AC}$, bem como nos conteúdos de Cálculo em várias variáveis (ALVES, 2011).

De modo particular, imprimimos, um viés de interpretação e exploração do gráfico de funções $f(z)$ na variável complexa; a localização das raízes de funções polinomiais, por intermédio do TFA; e, por fim; uma descrição pouco explorada nos compêndios especializados que resgata a ideia geométrica e dinâmica da noção de derivada e, consequentemente, o conceito de analiticidade de funções $f(z)$ na variável complexa (relacionada com as condições de Cauchy-Rieamann). Afirmamos, pois, que os softwares possibilitam enfatizarmos determinados aspectos e propriedades inexequíveis quando negligenciamos a tecnologia (ALVES, 2011). Com efeito, muitas das propriedades que indicamos aqui 
se relacionam com uma superfície descrita por $[x, y, u(x, y), v(x, y)] \in \mathbb{R}^{4}$, todavia, conseguimos vinculá-las a uma descrição num contexto dos espaços vetoriais, de dimensões dois e três.

Exemplificamos, por exemplo, quando as equações de Cauchy-Riemann, importantes no "estudo de analiticidade de funções" (SHOKRANIAN, 2011, p. 112), admitem uma interpretação gráficogeométrica que realiza o link conceitual com o comportamento de superfícies diferenciáveis no espaço tridimensional. Diferentemente dos livros de AC, que evidenciam seu caráter analítico e em outros assuntos como o Cálculo (ÁVILA, 2002), buscamos explorar uma interpretação gráfico-geométrica para as condições $(* *)$.

Discutimos, com apoio em Needham (2000), uma perspectiva de entendimento visual de propriedades e significação de conceitos matemáticos, do ponto de vista de sua ação e efeito (figuras 6 e 8). Nesses casos, recorremos ao significado envolvendo figuras mnemônicas que auxiliam a memória e o entendimento relativo ao processo de derivação de função $f(z)$, na variável complexa. Vale assinalar ainda métodos atuais desenvolvidos por especialistas com o intuito de visualizar conceitos em AC (ARNOLD \& ROGNESS, 2008).

\section{REFERÊNCIAS}

ALVES, Francisco. R. V. Aplicações da Sequência Fedathi na promoção das categorias do raciocínio intuitivo no Cálculo a Várias Variáveis. Tese (Doutorado em Educação) Universidade Federal do Ceará, Fortaleza, 2011, p. 353p. Disponível em: http://www.teses.ufc.br/tde_biblioteca/login.php

ALVES, Francisco R. V. (2012). Discussão da noção de integral imprópria com o auxílio do Geogebra. In: Conferência Latinoamericana de Geogebra, Montevidéu. 9-18. Disponível em: http://www.geogebra.org.uy/2012/home.php.

ALVES, Francisco. R. V. (2013a). Visualizing in Polar Coordinates with Geogebra. In: Geogebra International of Romania. p. 21-30. Disponível em: http://ggijro.wordpress.com/issues/vol-3-no-1/

ALVES, Francisco. R. V. (2013b). Exploring L'Hospital Rule with the Geogebra. In: Geogebra International of Romania. p. 15-20. Disponível em: http://ggijro.wordpress.com/issues/vol-3-no-1/

ALVES, Francisco. R. V. (2015). Visualização de Teoremas em Análise Complexa: exemplos no contexto da Transição Complexa do Cálculo. In: Revista Sinergia. v. 16, no 1, 65 - 76. Disponível em: http://www.cefetsp.br/edu/prp/sinergia/

ALVES, Francisco. R. V. Didática da Matemática: seus pressupostos de ordem epistemológica, metodológica e cognitiva. Revista Interfaces da Educação, v. 7, n 21, 131 - 150. 2016.

ALVES, Francisco. R. V. Engenharia didática com o tema integração de funções na variável complexa: análises preliminares, a priori e modelização de situações. Revista de Ensino de Ciências e Tecnologia em Revista. v. 7, no 1, 1- 15, 2017.

ARNOLD, Douglas, N. \& ROGNESS, Jonahtan. (2008). Möbius transformation revealed. In: Acta Mathematica, v. 55, no 10. p. 1226-1231. Disponível em: http://www.ima.umn.edu/ arnold//papers/moebius.pdf

ÁVILA, Geraldo. (2002). O ensino de Cálculo e da Análise. In: Matemática Universitária, no 33, p. 83-94. Disponível em: http://matematicauniversitaria.ime.usp.br/Conteudo/n33/n33_Artigo05.pdf 
NEEDHAM, Tristan. (2000). Visual Complex Analysis. Oxford: Oxfrod University Press.

NETO, Alcides, L. (1993). Funções de uma variável complexa. Rio de Janeiro: SBM.

PERES, Alicia. M. (2012). Visualizing complex solutions of polynomials (dissertation of Arts).

Austin: University of Texas. Disponível

http://repositories.lib.utexas.edu/bitstream/handle/2152/ETD-UT-2012-08-5996/PEREZ-MASTERSREPORT.pdf?sequence $=1$

SANTOS. Laudo. Claumir. (1998). Funções complexas de uma variável complexa: uma abordagem via software Mathematica. (dissertação de mestrado). Campinas: Universidade de campinas. $\quad$ Disponível em: http://www.bibliotecadigital.unicamp.br/document/?code=000133439\&fd=y.

SHOKRANIAN, Salahoddin. (2011). Introdução à Variável Complexa. Rio de Janeiro: Editora Moderna. 